\title{
Pathogenic variants in THSD4, encoding the ADAMTS-like 6 protein, predispose to inherited thoracic aortic aneurysm
}

\author{
Sandy Elbitar, PharmD, $\mathrm{PhD}^{1,2}$, Marjolijn Renard, $\mathrm{PhD}^{3}$, Pauline Arnaud, PharmD, PhD ${ }^{1,4,5}$, \\ Nadine Hanna, PharmD, PhD ${ }^{1,4,5}$, Marie-Paule Jacob, PhD ${ }^{1}$, Dong-Chuan Guo, PhD ${ }^{6}$, Ko Tsutsui, PhD ${ }^{7}$, \\ Marie-Sylvie Gross, MS ${ }^{1}$, Ketty Kessler, $\mathrm{PhD}^{8}$, Laurent Tosolini, $\mathrm{MS}^{1}$, Vincenzo Dattilo, $\mathrm{PhD}^{9}$, \\ Sebastien Dupont, $\mathrm{PhD}^{1}$, Jeremie Jonquet, $\mathrm{MS}^{1}$, Maud Langeois, $\mathrm{MS}^{5}$, Louise Benarroch, $\mathrm{PhD}^{1,10}$, \\ Melodie Aubart, MD, PhD ${ }^{1,11}$, Youmna Ghaleb, PharmD, PhD ${ }^{1,2}$, Yara Abou Khalil, PharmD ${ }^{1,2}$, \\ Mathilde Varret, PhD ${ }^{1}$, Petra El Khoury, PharmD, PhD ${ }^{1,2}$, Benoit Ho-Tin-Noé, PhD ${ }^{1}$, \\ Yves Alembik, MD ${ }^{12}$, Sébastien Gaertner, MD ${ }^{13}$, Bertrand Isidor, MD ${ }^{14}$, Laurent Gouya, MD, PhD ${ }^{5}$, \\ Olivier Milleron, MD5 ${ }^{5}$ Kiyotoshi Sekiguchi, $\mathrm{PhD}^{7}$, Dianna Milewicz, MD, $\mathrm{PhD}^{6}$, \\ Julie De Backer, MD, PhD ${ }^{3}$, Carine Le Goff, $\mathrm{PhD}^{1}$, Jean-Baptiste Michel, MD, PhD ${ }^{1}$, \\ Guillaume Jondeau, MD, PhD ${ }^{1,5}$, Lynn Y. Sakai, PhD ${ }^{15}$, Catherine Boileau, PharmD, PhD ${ }^{1,4,5}$ and \\ Marianne Abifadel, PharmD, PhD (1) ${ }^{1,2}$
}

Purpose: Thoracic aortic aneurysm and dissection (TAAD) is a life-threatening disease with often unrecognized inherited forms. We sought to identify novel pathogenic variants associated with autosomal dominant inheritance of TAAD.

Methods: We analyzed exome sequencing data from 35 French TAAD families and performed next-generation sequencing capture panel of genes in 1114 unrelated TAAD patients. Functional effects of pathogenic variants identified were validated in cell, tissue, and mouse models.

Results: We identified five functional variants in THSD4 of which two heterozygous variants lead to a premature termination codon. THSD4 encodes ADAMTSL6 (member of the ADAMTS/L superfamily), a microfibril-associated protein that promotes fibrillin-1 matrix assembly. The THSD4 variants studied lead to haploinsufficiency or impaired assembly of fibrillin-1 microfibrils. Ths $d 4^{+-}$mice showed progressive dilation of the thoracic aorta. Histologic examination of aortic samples from a patient carrying a THSD4 variant and from $T h s d 4^{+/-}$mice, revealed typical medial degeneration and diffuse disruption of extracellular matrix.

Conclusion: These findings highlight the role of ADAMTSL6 in aortic physiology and TAAD pathogenesis. They will improve TAAD management and help develop new targeted therapies.

Genetics in Medicine (2020) https://doi.org/10.1038/s41436-020-00947-4

Keywords: thoracic aortic aneurysm; THSD4; ADAMTSL6; diagnosis

\section{INTRODUCTION}

Thoracic aortic aneurysm leading to aortic rupture or dissection (TAAD) is an asymptomatic, life-threatening disease, with $50 \%$ mortality prior to arrival to hospital and $\sim 40 \%$ 30-day mortality for those admitted to hospital. ${ }^{1}$ In patients with TAAD, dilation of the arterial wall associated with a progressive loss of its ability to withstand the wall tension generated by high intraluminal pressure can lead to intramural or complete acute vessel wall rupture. ${ }^{2}$ Surgical treatment remains the main intervention to prevent dissections. ${ }^{3}$

While TAAD can occur in the context of syndromes, including Marfan syndrome (MFS; MIM 154700), Loeys-Dietz syndrome (MIM 609192, 608967, 610168, and

\footnotetext{
${ }^{1}$ Laboratory for Vascular Translational Science, INSERM U1148, Université de Paris, Centre Hospitalo-Universitaire Xavier Bichat, APHP, Paris, France; ${ }^{2}$ Laboratory of Biochemistry and Molecular Therapeutics, Faculty of Pharmacy, Pôle Technologie-Santé, Saint Joseph University of Beirut, Beirut, Lebanon; ${ }^{3}$ Center for Medical Genetics, Ghent University, Ghent, Belgium; ${ }^{4}$ Département de Génétique, Centre Hospitalo-Universitaire Xavier Bichat, APHP, Paris, France; ${ }^{5}$ Hospitalo-Universitaire Xavier Bichat, APHP, Centre de Référence Maladies Rares, Syndrome de Marfan et pathologies apparentées, Paris, France; ${ }^{6}$ Department of Internal Medicine, McGovern Medical School, University of Texas Health Science Center, Houston, TX, USA; ${ }^{7}$ Institute for Protein Research, Osaka University, Suita, Osaka, Japan; ${ }^{8}$ Centre for Evolution and Cancer, Division of Molecular Pathology, Division of Cancer Therapeutics, The Institute of Cancer Research, London, UK; ${ }^{9}$ IRCCS Istituto Centro San Giovanni di Dio Fatebenefratelli, Brescia, Italy; ${ }^{10}$ Inserm UMRS_974, Centre de recherche en myologie, G.H. Pitié-Salpétrière, APHP, Paris, France; ${ }^{11}$ Service de Neuropédiatrie, Hôpital Necker-Enfants-Malades, APHP, Paris, France; ${ }^{12}$ Department of Clinical Genetic, Hôpitaux Universitaires de Strasbourg, Strasbourg, France; ${ }^{13}$ Department of Hypertension, Vascular Diseases and Pharmacology, University of Strasbourg, Strasbourg, France; ${ }^{14}$ Service de Génétique Médicale, Hôpital Hôtel-Dieu, Centre Hospitalier Universitaire de Nantes, Nantes, France; ${ }^{15}$ Shriners Hospital for Children, Molecular \& Medical Genetics and Biochemistry \& Molecular Biology, Oregon Health \& Science University, Portland, OR, USA. Correspondence: Catherine Boileau (catherine.boileau@inserm.fr) or Marianne Abifadel (marianne.abi-fadel@inserm.fr)

These authors contributed equally: Sandy Elbitar, Marjolijn Renard

These authors contributed equally: Catherine Boileau, Marianne Abifadel
}

Submitted 3 June 2020; revised 11 August 2020; accepted: 11 August 2020

Published online: 28 August 2020 
610380) and aneurysms-osteoarthritis syndrome (MIM 613795), many cases are nonsyndromic. Importantly, up to $20 \%$ of individuals who experience nonsyndromic TAAD have a family history of TAAD (hTAAD, for heritable TAAD), ${ }^{2,4-6}$ suggesting that familial screening for genetic causes of TAAD could help identify individuals at risk for developing this condition.

Many genes have been identified that are associated with hTAAD. Alterations in these genes define three separate pathogenic mechanisms affecting the aortic wall: alteration of an extracellular matrix (ECM) component (such as fibrillin-1 ${ }^{7}$ encoded by $F B N 1$, or MAGP2 encoded by $M F A P 5^{8}$ ), alteration of TGF $\beta$ signaling (TGFBR $1,{ }^{9}$ TGFBR $2,{ }^{10}$ or $T G F B 2^{11}$ ), or alteration of smooth muscle cell (SMC) contraction (MYH11 encoding smooth muscle myosin heavy chain 11, or ACTA2 encoding smooth muscle a actin), metabolism or migration. ${ }^{12}$ HTAAD shows high clinical variability and low, as well as incomplete, penetrance. It tends to present in older patients, as highlighted by age-related onset of disease in almost all hTAAD family lineages. Furthermore, women tend to develop aortic manifestations at more advanced ages compared with men. ${ }^{13,14}$

To date, pathogenic variants in known genes account for less than $20 \%$ of hTAAD cases, suggesting that the genetic heterogeneity of this disorder is even greater than is currently understood. ${ }^{6,15}$ Given the importance of genetic screening for individuals at risk of developing hTAAD and the high proportion of cases of TAAD with unknown causes, we sought to identify novel genetic pathogenic variants associated with this condition. Here, we report five functional variants in the THSD4 (thrombospondin, type I, domain containing 4) gene in hTAAD families and probands with TAAD. THSD4 encodes ADAMTSL6, a microfibril-associated protein that binds directly to fibrillin-1 and promotes fibrillin-1 matrix assembly. ${ }^{16}$ It belongs to the ADAMTS/L superfamily, which comprises 19 secreted enzymes (ADAMTS, for A disintegrin and metalloproteinase with thrombospondin motifs) and seven secreted glycoproteins (ADAMTSL, for ADAMTS-like). ${ }^{17}$ The ADAMTS are extracellular metalloproteinases that are involved in ECM turnover. Members of the ADAMTSL subfamily are homologous to ADAMTS proteins, but do not have enzyme activity, as they lack the superfamily's consensus catalytic domain. ${ }^{16,18}$ Several studies conducted in mouse models of aortic dilation have recently reported that ADAMTS/L family members play a role in aortopathies, and their effects are often detected or enhanced by angiotensin II infusion. ${ }^{19-21}$ Members of this family would be interesting candidate genes in hTAAD. Our genetic approach links ADAMTSL6 to hTAAD. In vitro functional studies of the ADAMTSL6 variants confirmed their altered function. Histologic examination of aortic samples revealed diffuse disruption of the media. Furthermore, investigation of a $T h s d 4^{+/-}$mouse model showed pathogenic effects on the thoracic aorta. Thus, human genetic studies together with functional studies and a mouse model suggest that ADAMTSL6 is a new player in aortic physiology and pathology.

\section{MATERIALS AND METHODS}

\section{Ethics statement}

All patients gave their written informed consent for participation in this study in agreement with the requirements of French bioethics laws (institutional review board [IRB] approval from the Comité de Protection des Personnes [CPP] Ile de France XI, 78105 St Germain en Laye, registration number 11008).

\section{Patients}

Probands and families were recruited from the Centre National Maladies Rares-Syndrome de Marfan et apparentés, the French National Reference Centre located at Bichat Hospital (Paris, France) and through competence centers nationwide. Genomic DNA was isolated from peripheral blood leukocytes by standard procedures as previously reported. ${ }^{8,11,15}$ In total, 1114 French probands were screened for diagnosis of possible syndromic (notably MFS) or nonsyndromic TAAD through a multidisciplinary clinical assessment process including systematic slit-lamp examination, extensive imaging (including aortic echocardiography), and molecular analysis.

\section{Exome sequencing}

It was performed on 106 patients from 35 TAAD-affected families in which no causal variant in genes previously associated with TAAD had been identified (consecutive to Sanger sequencing or next-generation sequencing capture panel of candidate genes). DNA samples from affected members of TAAD families were sequenced by Integragen S.A. (Evry, France), as previously reported. ${ }^{8,11}$

\section{DNA amplification and variant detection}

Genomic DNA was sheared into fragments ranging from 200-300 bp using a Bioruptor ultrasonicator (Diagenode, Seraing, Belgium). A custom capture array (NimbleGen SeqCap ${ }^{\circledR}$, Roche) was designed and used to capture all coding exons, splice sites, and the flanking intron sequences of THSD4 gene (NM_024817) and 23 other genes already known to be associated with Marfan syndrome and associated disease and heritable thoracic aneurysm and dissections. Amplification, sequencing, and variant annotation were performed as previously described. ${ }^{8,11,15}$ All variants of interest were systematically confirmed by bidirectional Sanger sequencing primers (in Supplementary table S1).

Additional details concerning materials and methods are found in the Supplementary Information.

\section{RESULTS}

\section{Next-generation sequencing allowed the identification of TAAD-associated genetic variants}

To identify the genetic causes of TAAD in a French cohort, we performed sequencing of 1114 unrelated patients with a clinical spectrum ranging from syndromic to nonsyndromic TAAD. We also analyzed exome sequencing data from 35 French hTAAD families, investigating novel or rare variants 
(minor allele frequency [MAF] $<0.1 \%$ in non-Finnish European populations, according to gnomAD). Overall, we found a likely pathogenic variant (class 4 and 5 according to American College of Medical Genetics and GenomicsAssociation for Molecular Pathology [ACMG-AMP] recommendation) in $\sim 30 \%$ of the cases, a class 3 variant (VUS) in $\sim 5 \%$ of the cases, and in $\sim 65 \%$ of the cases, no interesting potential pathogenic variant was identified in previously identified genes. In the context of identifying new genes associated to TAAD, we pinpointed 22 variants in a new candidate gene, THSD4, that encodes ADAMTSL6, an ECM protein known to promote fibrillin-1 matrix assembly (Fig. 1a, Table 1, and Supplementary table S2). THSD4 is composed of 17 coding exons, with two transcript variants encoding two isoforms of 1018 and 658 amino acids, respectively (Fig. 1b). Five of the 22 variants were found in probands for whom family members were available for segregation analysis (Fig. 1a and Table 1) and thus were further investigated.

Two heterozygous variants were found in two probands. Both variants resulted in a premature termination codon (PTC) and have not been previously reported in population databases. The male proband (II.6) of the first family (TAA1889; Fig. 1a and Table 1) suffered from type A aortic dissection at the age of 43 . The aortic diameter prior to dissection was unknown. He exhibited a mild pectus excavatum, dolichostenomelia, and a high-arched palate. He was not known to have arterial hypertension. He carried the c.740del variant, a deletion of one of the three consecutive thymines at position 738 , leading to the p.(Leu247*) PTC variant. His mother (I.2), operated on for ascending aortic dissection at the age of 75 years (aortic diameter unknown), also carried the variant. The proband's two sons (III.5 and III.6), aged 24 and 25 years, respectively, did not present dilated aortas upon echocardiography and did not carry the variant. Finally, the proband's brother (II.2; 61 years) did not carry the variant, and had a normal aortic root diameter but mild dilation of the ascending tubular aorta, with a history of arterial hypertension.

The second PTC variant was found in a family (TAA-1839; Fig. 1a and Table 1). The molecular event is a singlenucleotide deletion in a three-guanine repeat at position 1400 (c.1402del) that leads to a PTC (p.[Ala468Glnfs*45]). The variant was identified in the female proband (II.2) and her daughter (III.3). Both displayed dilation of the ascending aorta, with diameters of $42 \mathrm{~mm}$ at the aortic root and $47 \mathrm{~mm}$ at ascending aorta for the mother and $28 \mathrm{~mm}$ at the aortic root and $32 \mathrm{~mm}$ at the ascending aorta for the daughter. The proband's sister (II.3) (aortic root: $33 \mathrm{~mm}$, ascending aorta: $36 \mathrm{~mm}$ ) also carried the variant. The proband's mother (I.2) had a normal aortic root diameter $(37 \mathrm{~mm})$ at the age of 75 years, and did not carry the variant. The proband inherited the variant from her father (I.1), for whom there is no evidence of the disease, and can constitute a case of incomplete penetrance, especially because exome sequencing performed in this family did not allow identification of any other possible gene variant linked to the disease.
Furthermore, a 9-bp in-phase duplication that has not been previously reported in population databases was identified in a third family (TAA-1850; Fig. 1a, Table 1). The c.137_145dup duplication is expected to lead to p. (Asp46_Gly48dup). Aortic root dilation (48 mm) was discovered in the proband (II.3) after myocardial infarction at the age of 39 years. He suffered from high blood pressure and hypercholesterolemia, and was an active smoker. He has three children (III.1, III.2, and III.3) without aortic dilation and who did not carry the duplication. His brother (II.4), who carried the duplication, was operated on for aortic dissection at the age of 42 years, and dilation of the ascending aorta was present prior to dissection. Finally, the proband's father (I.1), who carried the duplication, was operated on for aortic dilation of the ascending aorta and abdominal aorta at the age of 68 years.

Two heterozygous missense variants were identified in the series of unrelated probands tested: substitutions in exon 5 (c.961T>A; p.[Tyr321Asn]) and in exon 13 (c.2341C>T; p. [Arg781Trp]). The tyrosine substitution was found in a female proband who underwent Bentall surgery for aortic regurgitation at the age of 61 years. Her daughter was the only other family member available for study. The daughter had an aortic root diameter of $30 \mathrm{~mm}$ and ascending aorta diameter of $29 \mathrm{~mm}$ at the age of 25 years, and carried the variant. This variant has not been previously reported in population databases. It was predicted by PolyPhen-2 to be probably damaging, by SIFT to be deleterious, and by the CADD algorithm to be among the top $1 \%$ of the most deleterious substitutions possible in the human genome (score of 29.1). The p.(Arg781Trp) variant was found in a proband who had elective surgery for an aortic root diameter of $50 \mathrm{~mm}$ at 57 years old. His brother, who does not carry the variant, is alive at the age of 84 years, with no evidence of a thoracic aortic disease. This variant is rare with a reported frequency of 0.000035 in gnomAD European non-Finnish database (rs767807136). It was predicted to be probably damaging by PolyPhen-2, to be deleterious by SIFT, and to have a CADD score of 21.0 , indicating that it is in the top $1 \%$ of most deleterious substitutions possible in the human genome.

We selected a sixth variant from among 17 other rare variants (frequency $<0.1 \%$ in the gnomAD European nonFinnish database) identified by sequencing the THSD4 gene in the unrelated probands with TAAD (Supplementary table S2). This variant, p.(Gly753Asp) (c.2258G $>$ A), was found in three unrelated TAAD probands. Although this variant was also found in gnomAD controls, its frequency is low (0.00035 [45/ $128,528]$ in the gnomAD European Non-Finnish database). Furthermore, it affects a $\mathrm{CpG}$ doublet, which is known to be highly mutable. We therefore hypothesized that this could represent either a founder pathogenic variant or a variant hotspot for this disease and we included this variant in our functional studies. The locations of these six variants with respect to the gene and the protein are shown in Fig. 1b. It should be noted that three of the variants affect both the type 1 and type 2 isoforms of ADAMTSL6. 


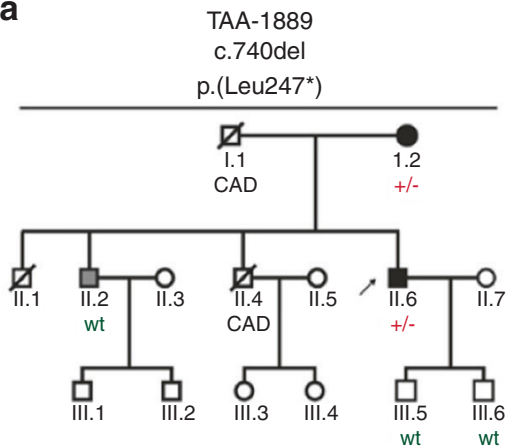

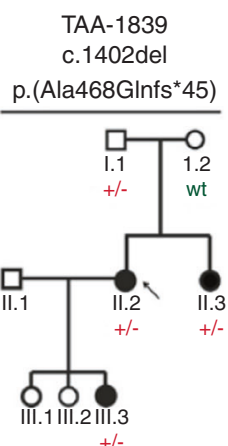

$+/-$
TAA-1850

c. $137 \_145$ dup

p.(Asp46_Gly48dup)

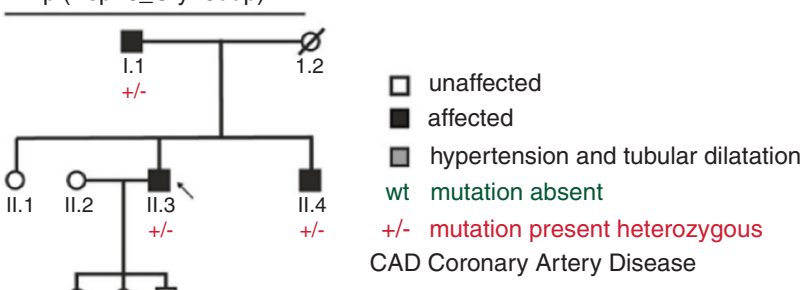

wt wt wt

b

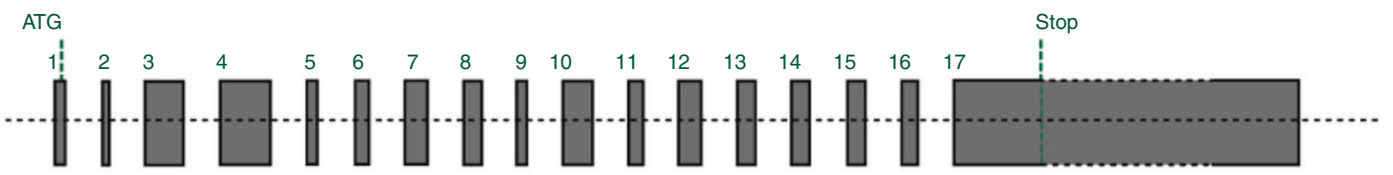

Number of amino acids

10

155

$\begin{array}{lllllllllllll}304 & 339 & 384 & 453 & 511 & 544 & 636 & 679 & 747 & 806 & 863 & 923 & 972\end{array}$

1018

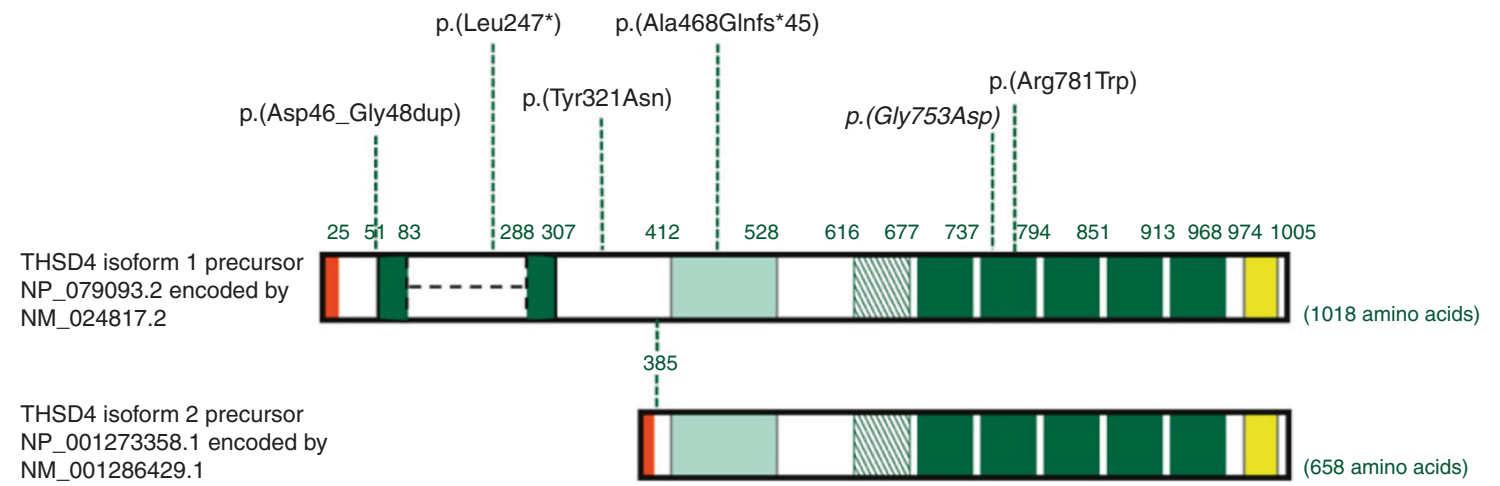

SP: Signal Peptide

ADAM-TS Spacer

TSP1: Thrombospondin type 1 repeats

Probable additional TSP1 repeat

PLAC domain

Fig. 1 THSD4 functional variant segregation in families with a history of thoracic aortic aneurysms. (a) Segregation of THSD4 variants $p$. (Leu247*), p.(Ala468GInfs*45), and p.(Asp46_Gly48dup) with symptoms of cardiac disease respectively in families TAA-1889, TAA-1839, TAA-1850 with a history of thoracic aortic aneurysm and dissection (TAAD). Black arrows indicate probands. (b) Schematic representation of the THSD4 gene and protein indicating the location of pathogenic variants identified in French families. THSD4 is composed of 17 coding exons. Two premature termination codon (PTC) variants were identified in two families: c.740del in exon 4 leading to p.(Leu247*) and c.1402del in exon 8 leading to p.(Ala468Glnfs*45). The variant p. (Asp46_Gly48dup) was identified in a third family. Functional studies were performed for the following missense variants: p.(Tyr321Asn), p.(Gly753Asp), and p.(Arg781Trp). Reference transcript variants 1 and 2 are shown: variant 1 (NM_024817.2) represents the longer transcript and encodes the longer isoform (NP_079093.2). Variant 2 (NM_001286429.1) differs in the 5' UTR and lacks multiple 5' coding exons, compared with variant 1. It represents use of an alternate promoter and initiates translation from an alternate start codon. The encoded isoform 2 (NP_001273358.1) has a distinct N-terminus and is shorter than isoform 1. 


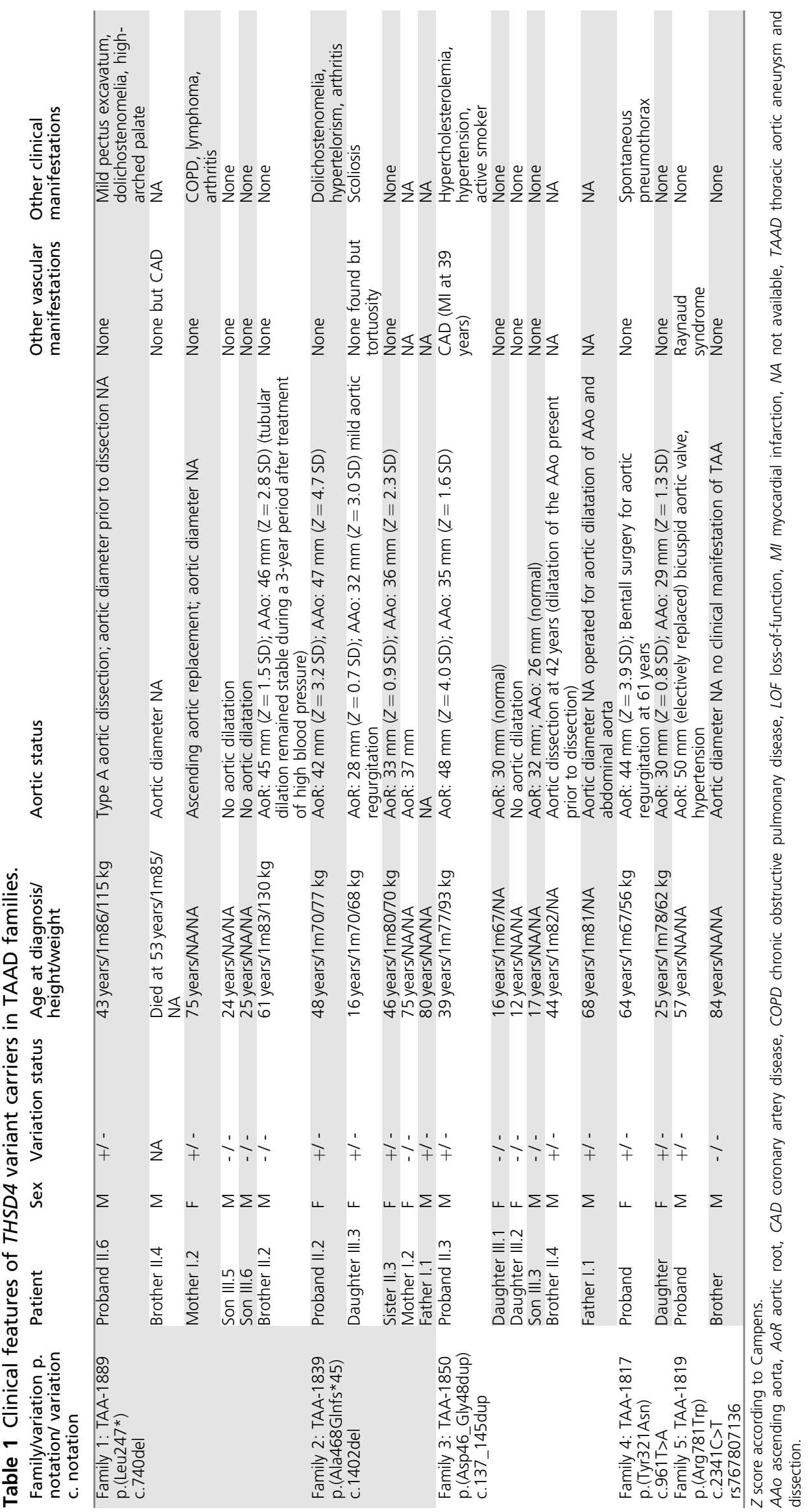


PTC and missense variants in THSD4 lead to haploinsufficiency or impaired assembly of fibrillin-1 microfibrils

We next sought to investigate the functional effect of the DNA variants identified as described above. For the PTC variants, THSD4 messenger RNA (mRNA) expression was tested in cultured skin fibroblasts from four patients with and without emetine, which prevents nonsense-mediated mRNA decay (NMD). Basal expression varied greatly between individuals, even between patients carrying the same THSD4 variation, and this effect was not related to age (Fig. 2a). THSD4 expression was significantly increased in the presence of emetine, demonstrating NMD both for the p.(Leu247*) variant in patient (II.6) from the TAA-1889 family and the $\mathrm{p}$. (Ala468Glnfs*45) variant in patients (III.3, II.2, and I.1) from the TAA-1839 family (Fig. 2a). Thus, the functional effects of these PTC variants could be explained by haploinsufficiency. However, to determine the effects of the three THSD4 missense variants, MG-63 cells were transiently transfected with wild-type (WT) or mutated constructs, knowing that ADAMTSL6 is an ECM protein that promotes fibrillin-1 microfibrils assembly in those cells. When cells were transfected with the WT construct, an extensive and wellorganized fibrillin-1-positive microfibril array formed in five days compared with empty vector (Fig. $\mathbf{2 b , c}$ ). In contrast, cells transfected with the THSD4-Arg781Trp or THSD4Tyr321Asn constructs exhibited very few fibrillin-1-positive microfibrils (Fig. 2d,e). In cells transfected with the THSD4Gly753Asp construct, few islands of fibrillin-1-positive microfibrils were found; but in general, only punctate nonorganized structures were observed (Fig. 2f). Semiquantitative analysis of the images obtained from the immunocytochemistry analysis showed that there was a significant decrease in the signal obtained with the THSD4-Arg781Trp and THSD4-Tyr321Asn constructs compared with WT (Fig. 2g), which confirmed the qualitative observations. Cells transfected with the THSD4-Gly753Asp construct exhibited a strong cell staining of fibrillin-1 compared with WT. Taken together, these findings suggest that missense variants in THSD4 lead to impaired assembly of fibrillin-1 microfibrils (Fig. 2, Supplementary Fig. S1).

\section{THSD4 variant is associated with disorganization of the aortic media}

The human aortic pathology associated with THSD4 pathogenic variants was assessed using the only available patient's aortic tissue, i.e., from the 48-year-old female proband carrying the THSD4 p.(Ala468Glnfs*45) PTC variant. Aortic tissue from a female patient carrying the p.(Cys2307Arg) variant in the FBN1 gene, one of the major genes in hTAAD, was used as reference for the aortic histology of hTAAD and compared with normal ascending aorta tissue from a healthy control. Pathology typical of thoracic aortic disease was found in both patients, including disorganization of the media with fragmentation and disarray of elastic fibers, accumulation of proteoglycans, and areas of medial degeneration along with
SMC rarefaction (Fig. 3a-c). TGF $\beta 1$ enrichment was observed, particularly around microvessels, compared with normal aorta (Fig. 3d), highlighting the angiogenic process associated with TAAD. ${ }^{22}$ Aneurysmal tissues showed an increase in nuclear phosphorylated SMAD2/3 levels in the most affected part of the medial layer that did not colocalize with TGF $\beta 1$ (Fig. 3e), as previously reported. ${ }^{23}$ Fibrillin-1 was almost undetectable in healthy aortic tissue (Fig. 3f), presumably because of poor immunoavailability due to its tight incorporation within hydrophobic elastic fibers. ${ }^{24,25}$ It was organized in aggregates that accumulated parallel to elastic fibers and exhibited a pericellular signal in aortic tissue from variants carriers (Fig. 3f). In agreement with previous results, ${ }^{26}$ fibronectin seemed to be more abundant in aneurysmal tissues compared with healthy aorta (Fig. 3g). Aortic sections from the FBN1 p.(Cys2307Arg) and THSD4 p. (Ala468Glnfs*45) carriers showed condensed pericellular fibronectin aggregated around SMCs (Fig. 3g). These findings showed medial degeneration with diffuse disruption of ECM and activation of TGF- $\beta$ pathway associated with the THSD4 PTC variant.

\section{Thsd $^{+/-}$mice present progressive thoracic aortic dilation}

To confirm observations found in human aortas and to identify the role of ADAMTSL6 encoded by THSD4 in aortic dilation, a mouse model was generated. Ths $d 4^{+/-}$mice had a normal lifespan. Vascular echography analysis was performed to assess thoracic aortic diameters in $\mathrm{WT}$ and $T h s d 4^{+/-}$mice at different ages (3-12 months). We observed a significant increase in aortic diameter in the Ths $4^{+/-}$mice $\left(P<1.10^{-4}\right.$ at 6, 8, and 12 months) compared with WT (Fig. 4a). The aortic diameters significantly increased with time (sinus of Valsalva $P<1.10^{-4}$; ascending aorta $P<1.10^{-4}$; arch $P<1.10^{-3}$; descending aorta $P<1.10^{-2}$ ) in both WT and Thsd $4^{+/-}$mice (Fig. 4b). A statistically significant interaction between genotype and time was observed at the sinus of Valsalva $(P=0.01)$, arch $(P=0.02)$, and descending aorta $\left(P<1.10^{-3}\right)$. This interaction demonstrated that aortic diameter in Ths $d 4^{+/-}$mice increased more with time than in WT mice. To test whether there are differences between aortic tissue from WT and Thsd $4^{+/-}$mice, we performed histochemical analysis. Young Ths $4^{+/-}$mice (0-5 months) do not seem to have a defect in elastic fiber formation. The aortas of $T h s d 4^{+/-}$ mice at 6 and 9 months showed patchy accumulation of Alcian Blue-positive areas, revealing the presence of proteoglycans, unlike those of a 6-month-old WT mouse (Fig. 4c). This observation was confirmed by a more pronounced disorganization of autofluorescent elastin in $T h s d 4^{+/-}$compared with WT mice (Fig. 4d). The nuclei of Ths $d 4^{+/-}$mice exhibited a more significant degree of DNA degradation compared with WT mice at 6 months of age, as indicated by the ratio of TUNEL-positive to Dapi-positive nuclei (Fig. 4e). Interestingly, visualization of nuclei in the same area of an aorta tissue sample taken from a 9-month-old Ths $d 4^{+/-}$ mouse by three different methods, revealed that, while some nuclei were predominantly positive for hematoxylin staining 
a

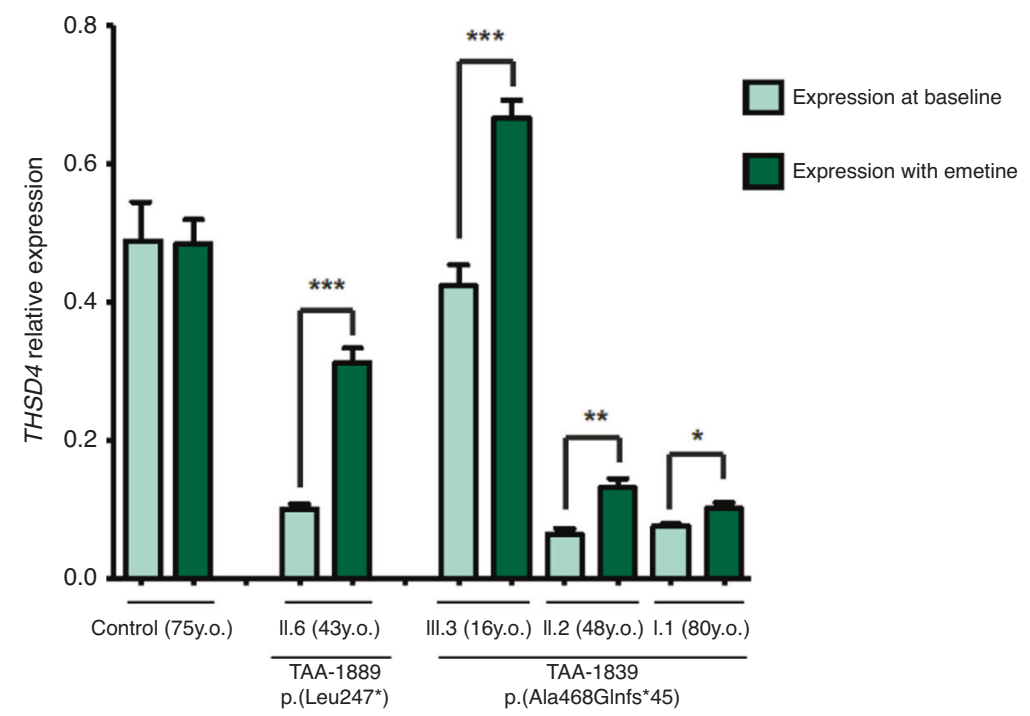

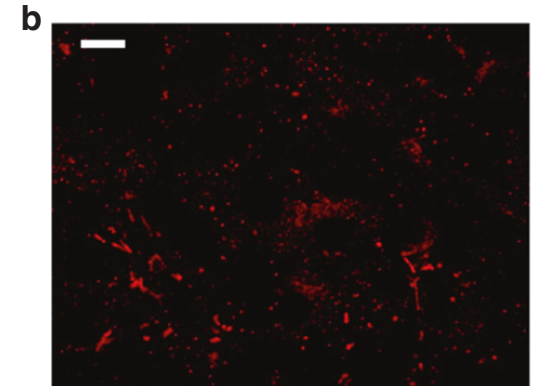

Empty vector

d

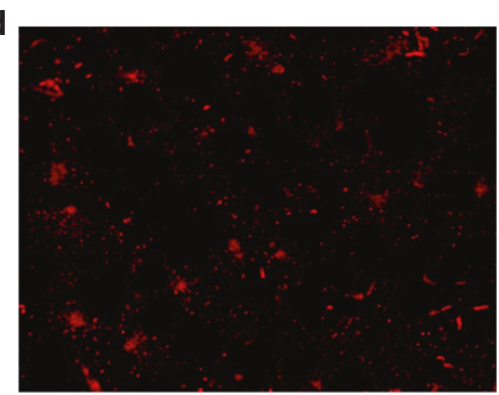

p.(Arg781Trp)

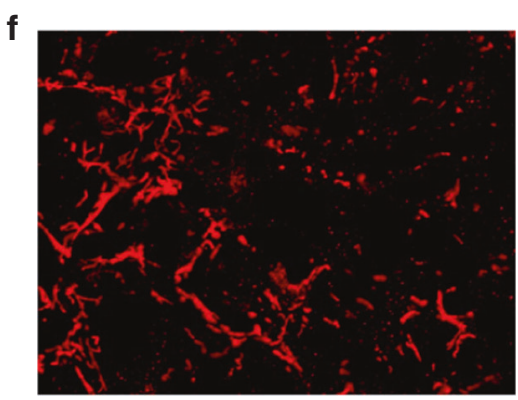

p.(Gly753Asp)

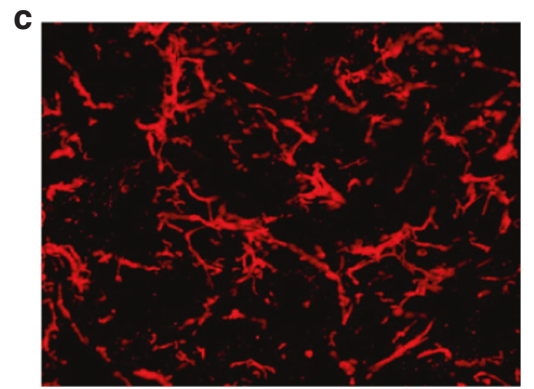

Wild type (WT)

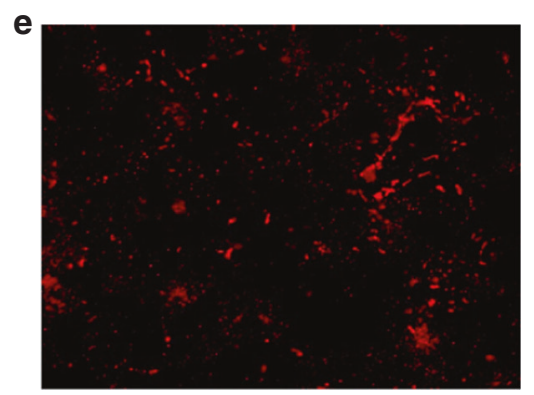

p.(Tyr321Asn)

g

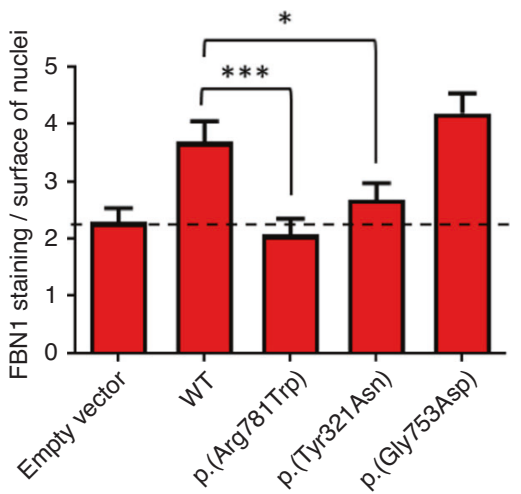


Fig. 2 Missense variants in THSD4 lead to haploinsufficiency or impaired assembly of fibrillin-1 microfibrils. (a) Evaluation of nonsense-mediated messenger RNA (mRNA) decay (NMD) in skin fibroblasts from THSD4 premature termination codon (PTC) variants carriers. Skin fibroblasts were obtained from the proband (II.6) carrying the p.(Leu247*) variant in TAA-1889 and patients (III.3, II.2, and I.1) carrying the p.(Ala468GInfs*45) variant from family TAA-1839. Fibroblasts from individual 1.2 from family TAA-1839 were used as the control. Fibroblasts were grown with or without emetine (an inhibitor of the NMD pathway). Quantitative reverse transcription polymerase chain reaction (RT-qPCR) was performed to evaluate the basal level of expression of THSD4 in those conditions. RT-qPCR data are presented as means \pm standard error of the mean $(n=6$ for variant carriers, $n=3$ for control, experiment repeated on two independent cell cultures). Statistical differences were analyzed using a two-tailed Mann-Whitney test $\left({ }^{*} P<0.05 ; * \star P<0.01 ; * \star * P<0.001\right)$. (b-f) Immunocytochemistry of fibrillin-1 (red) was performed in MG-63 cells transfected with empty vector (b) or vectors encoding wild-type (WT) ADAMTSL6 (c) or the different mutated forms (p.[Arg781Trp] [d], p.[Tyr321Asn] [e] and p.(Gly753Asp) [f]) 5 days after plating. Images are representative of three independent experiments. Scale bar for $(\mathbf{a}-\mathbf{e}), 20 \mu \mathrm{m}$. (g) A semiquantitative assay was performed using fibrillin-1 staining normalized to the nuclear surface, in images obtained from the immunocytochemistry analysis 5 days after plating. Results are expressed as means of normalized fibrillin- 1 staining \pm standard error of the mean of 25 images per condition (WT was analyzed in duplicate). The dashed line indicates the mean level of fibrillin-1 staining normalized to surface of nuclei, obtained with empty vector. Statistical differences were analyzed using a two-tailed Mann-Whitney test $\left({ }^{*} P<0.05 ; * \star \star P<0.001\right)$.

(tinctorial), they were negative for Dapi staining (double strand DNA marker) but positive for TUNEL staining (degraded DNA marker) (Fig. 4f). This suggests that there is a decrease in the number of SMC nuclei present in the 9month-old Ths $d 4^{+/-}$mouse, implying a loss of SMC tissue in the $T h s d 4^{+/-}$mice as they age. Therefore, accumulation of proteoglycans with disorganization of elastic fibers in the ECM along with SMC alteration were observed in the Ths $d 4^{+/-}$mice, and could explain the aortic dilation observed in these mice as they age compared with WT.

\section{DISCUSSION}

In this study, we identified loss-of-function pathogenic variants in the THSD4 gene encoding ADAMTSL6 as a novel mechanism underlying inherited thoracic aortic aneurysm in humans. THSD4 encodes ADAMTSL6, a microfibrilassociated protein known to bind directly to fibrillin-1 and to promote fibrillin-1 matrix assembly. ${ }^{16}$ We showed that PTC and missense variants in THSD4 lead to haploinsufficiency of ADAMTSL6 and/or impaired assembly of fibrillin-1 microfibrils. Furthermore, the observed correlation of these variants with the disease in patients was confirmed by a Ths $d 4^{+/-}$mouse model that showed progressive aortic dilation at the level of the thoracic aorta that increased significantly over time compared with control mice. All these data in patients and mice support that THSD4 is a novel protagonist in the pathophysiology of hTAAD and confirm the role of ADAMTSL6 in microfibril formation and homeostasis. ${ }^{16}$

ADAMTSL6 is one of the ADAMTSL proteins, a subgroup of the ADAMTS superfamily. ${ }^{16}$ ADAMTS-1, ADAMTS-4, and ADAMTS-5 are proteoglycanases that cleave large hyaluronan-binding proteoglycans in the vasculature. ${ }^{27,28}$ Pathogenic variants in ADAMTSL proteins have been implicated in two human genetic diseases: bone geleophysic dysplasia for ADAMTSL2 $2^{29}$ and autosomal recessive familial ectopia lentis for ADAMTSL4. ${ }^{30}$ No ADAMTS/L variants have previously been associated with thoracic aortic disease in humans except a single recent report $^{31}$ of a patient with geleophysic dysplasia carrying a pathogenic variant in ADAMTSL2. ${ }^{29}$ At 48 years of age, this is the oldest patient ever reported presenting with the disease. Interestingly, along with typical skeletal features, the patient displayed a severely regurgitant aortic valve that was surgically replaced, with a dilation of the ascending aorta and the main pulmonary artery. Since the mean life expectancy for these patients is in the third decade, it is possible that the aortic pathology of this autosomal recessive form of the disease appears only at midlife and thus had never been observed before this case. In keeping with this hypothesis, all of the probands carrying THSD4 variants assessed in this study were diagnosed with TAAD in midlife. Therefore, pathogenic variants in these two genes can be associated with a later onset TAAD.

Even though the exact function of ADAMTSL2 remains incompletely elucidated, it is known to interact directly with the C-terminal and the N-terminal half of fibrillin- $1^{32}$ and to partner with TGF $\beta$ binding protein $1 .^{17,29,33-35}$ In human as well as in mouse, ADAMTSL6 is known to promote microfibril assembly and in mouse it binds to the Nterminal half of fibrillin-1. ${ }^{16,36}$ This could confirm that ADAMTSL2 and ADAMTSL6 play physiological roles in the development and maintenance of the aortic ECM as well as pathological processes, like fibrillin-1 and TGF $\beta$ signaling, also known to be altered in hTAAD. ${ }^{31,37,38}$ Indeed, histological analysis of mouse and human aortic tissues showed that THSD4 variants led to the same medial degeneration as observed with pathogenic variants within the FBN1 gene as well as in several genes encoding proteins of TGF $\beta$ metabolism and signaling pathway. ${ }^{16,36}$ Furthermore, pericellular fibronectin aggregated around SMCs was also observed. Fibronectin acts not only as a general orchestrator of matrix assembly, but is also a matrix stabilizer since it is essential for maintenance of cell-matrix adhesion sites. ${ }^{32}$ Fibronectin fibrillogenesis is typically pericellular, involves interactions with the cell surface and clustering of $\alpha 5 \beta 1$ integrins, and has been shown to be a prerequisite of microfibril formation, ${ }^{39}$ interacting with the C-terminus of fibrillin- $1 .^{40}$ In keeping with these data, it could be speculated that haploinsufficiency for ADAMTSL6 in the aortic wall alters microfibrillar homeostasis, triggering a healing process that initiates through fibronectin production and fibrillogenesis but that is stalled at the stage of pericellular fibrillin-1-rich microfibril assembly. Therefore, the signals of altered homeostasis are maintained and could explain the abundance of pericellular fibronectin observed in the aortic tissue obtained from the 
a Healthy aorta

щ

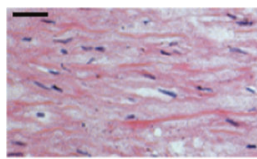

b
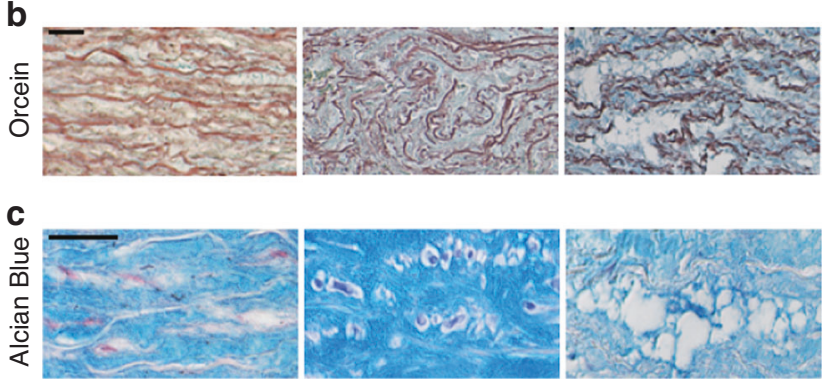

d

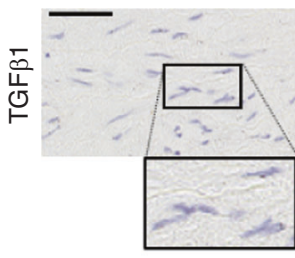

e

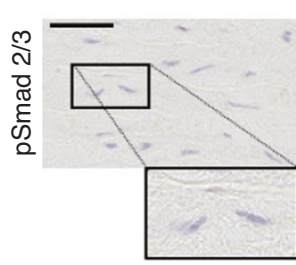

THSD 4 p.(Ala468Glnfs*45)
FBN1 p.(Cys2307Arg)
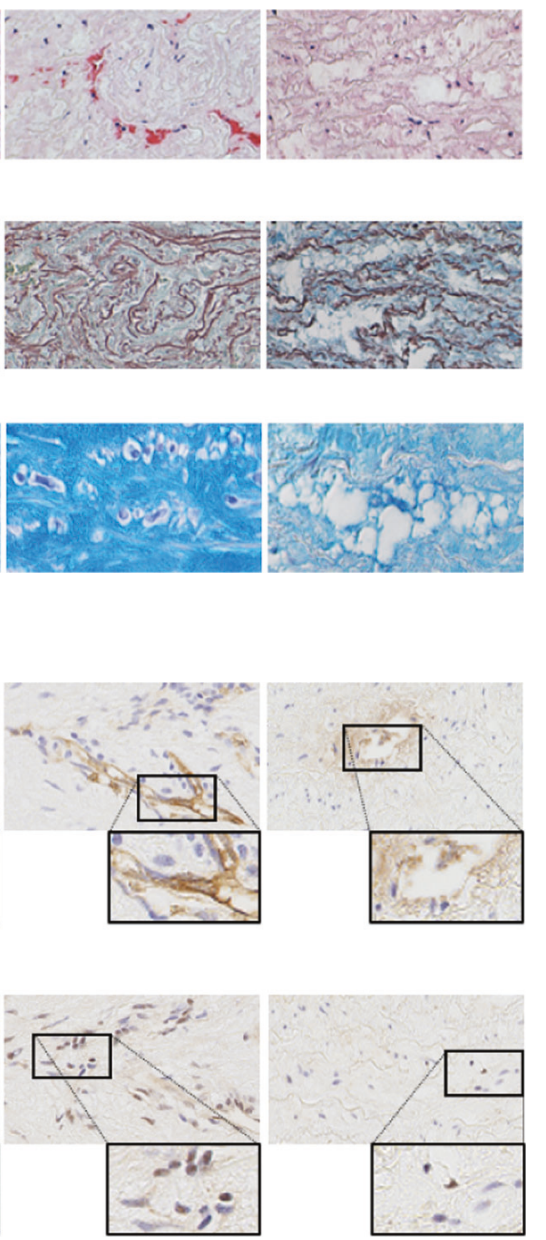

f
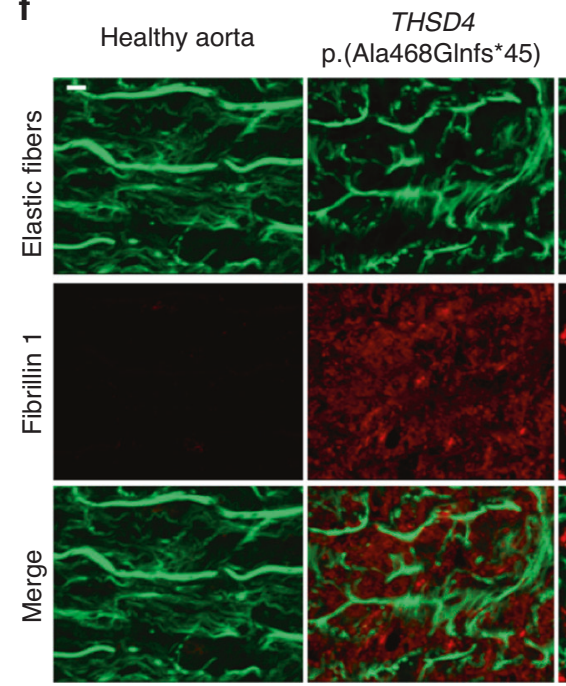

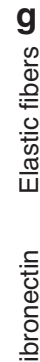
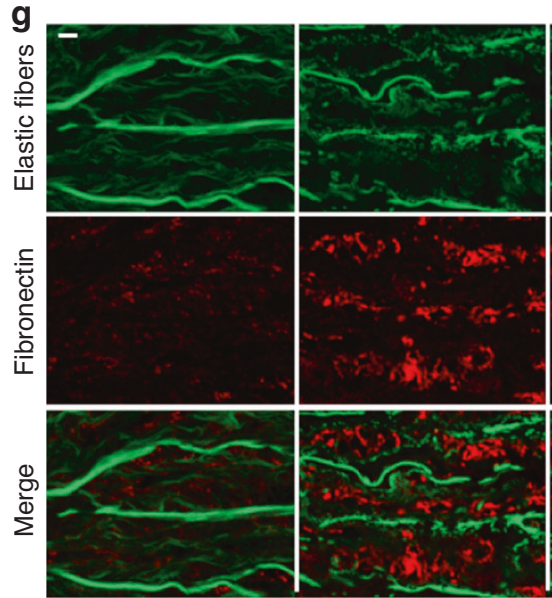

FBN1 p.(Cys2307Arg)
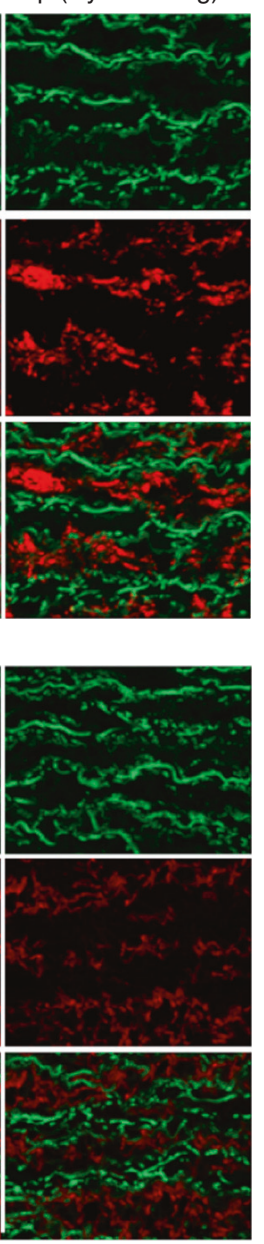

Fig. 3 A pathogenic premature termination codon (PTC) variant in THSD4 is associated with medial degeneration and activation of the TGF $\beta$ pathway. (a-c) Histology of aortic media from the 48-year-old TAA-1839 proband (II:2), carrying the THSD4 p.(Ala468Glnfs*45) variant (middle) compared with a 54-year-old thoracic aortic aneurysm (TAA) female patient carrying the FBN1 p.(Cys2307Arg) variant (right) along with normal ascending aorta obtained from a 52-year-old female organ donor (left). Staining with hematoxylin-eosin (HE) (a), orcein (b), and Alcian Blue (c) revealed the characteristic features of medial degeneration: disorganization of the media with fragmented and disordered elastic fibers, accumulation of proteoglycans and smooth muscle cell (SMC) rarefaction in the individuals with the THSD4 and FBN1 variants. Scale bar, $25 \mu \mathrm{m}$. (d) Immunohistochemical analysis of the same tissues showed increased TGF $\beta 1$ expression, particularly around microvessels, in aortic tissue from the THSD4 and FBN1 variant carriers compared with normal aorta. Scale bar, $50 \mu \mathrm{m}$. (e) Aneurysmal tissue from individuals carrying THSD4 and FBN1 variants show a diffuse increase in nuclear pSmad2/3 expression in the most affected medial layer compared with control aortic tissue. Scale bar, $50 \mu \mathrm{m}$. (f) Immunofluorescent (red) fibrillin-1 is almost undetectable in healthy aorta. It appears as discontinuous, aggregated microfibrils parallel to elastic fibers in aortic tissue from the patients carrying the FBN1 p.(Cys2307Arg) and THSD4 p.(Ala468GInfs*45) variants. Scale bar, $10 \mu \mathrm{m}$. (g) Fibronectin appears to be overexpressed in aneurysmal tissues compared with healthy aorta. The aneurysmal aortic sections showed condensed, pericellular fibronectin staining with some aggregates appearing around SMCs. Scale bar, $10 \mu m$. Elastic fibers appear in green in (f, g) (autofluorescence).

patient carrying the THSD4 p.(Ala468Glnfs*45) PTC variant. The presence in the same location of microfibrils could also reveal a role of ADAMTSL6 in promoting higher level organization of the microfibrillar network. Thus, Thsd4 haploinsufficiency may lead to improper orchestration and orientation of microfibril assembly. Further studies are needed to test this hypothesis and notably a possible interaction of ADAMTSL6 and fibronectin.

Another typical aspect of TAAD aortic pathology observed in a patient's samples was the presence of TGF $\beta$ in the media and the presence of $\mathrm{pSmad} 2 / 3$ in SMC nuclei. Adamtsl6 directly binds TGF $\beta$, and administration of Adamtsl6 suppresses nuclear localization of pSmad2/3 in transgenic Fbn $1 \mathrm{mgR} / \mathrm{mgR}$ mice. $^{36}$ Therefore, the higher TGF $\beta$ signal observed for the THSD4 variant carrier may be related to the lack of available ADAMTSL6 in the aortic wall.

Our findings confirm that increased expression of THSD4 promotes the assembly of fibrillin-1 microfibrils and show that missense variants lead to their impaired assembly. This observation is in accordance with previous results showing that fibrillin-1 matrix assembly was promoted in the early but not the late stage of the assembly process when cells were 


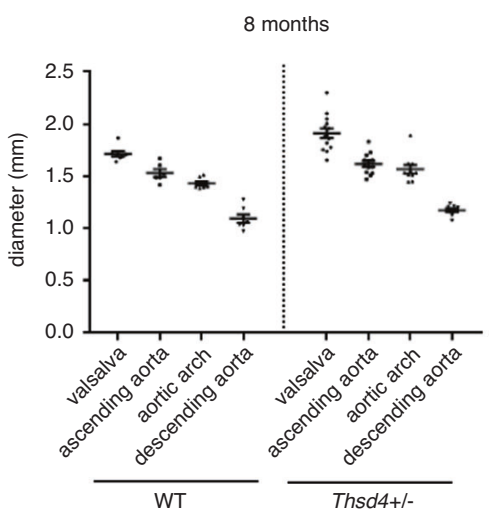

b

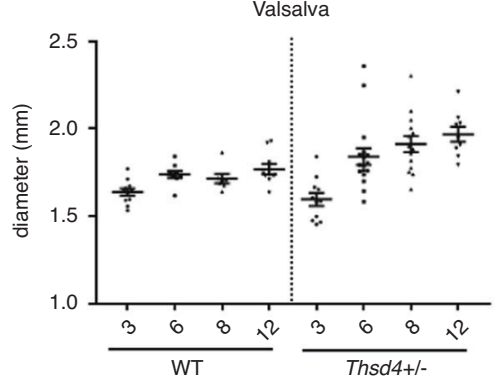

12 months
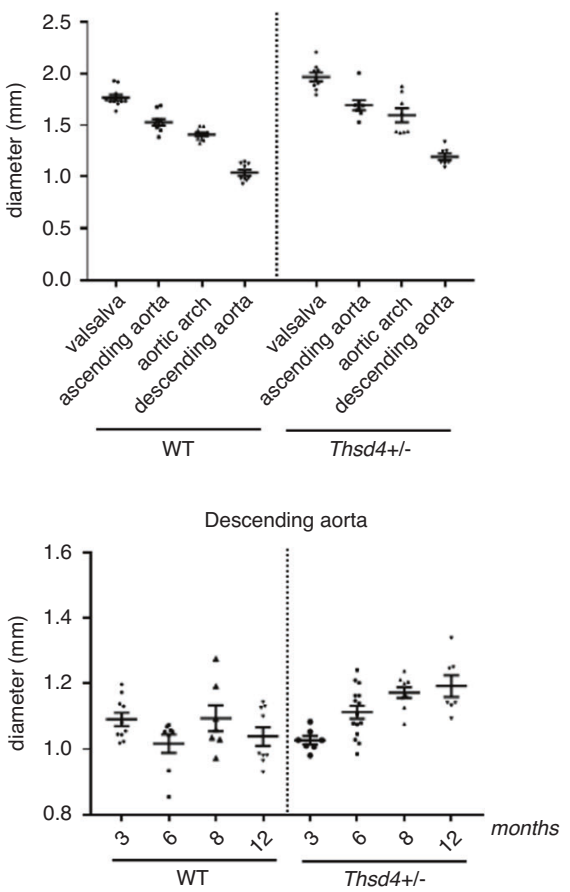

C Thsd4 wt (6 months)

Thsd4 +/- (6 months) Thsd4 +/- (9 months)
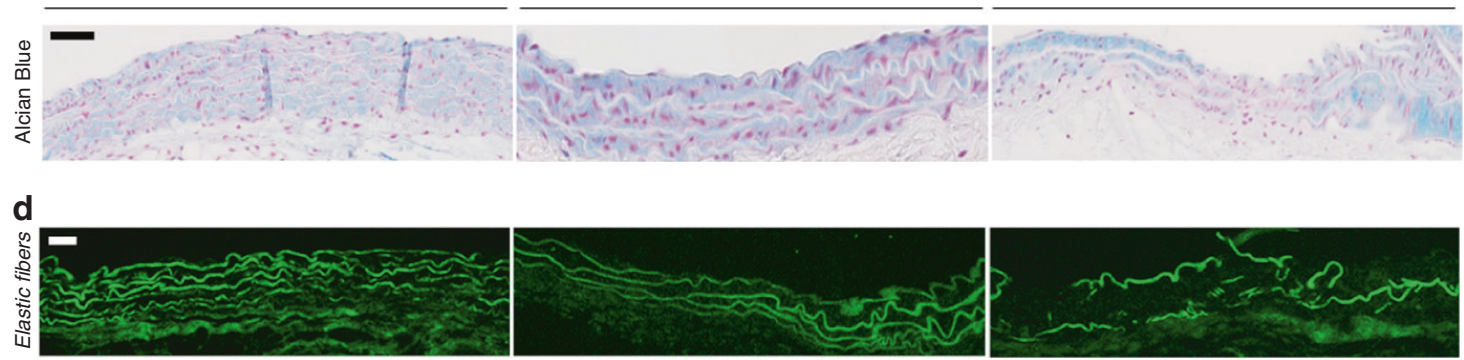

e

Thsd4 wt (6 months)

Thsd4 +/- (6 months)
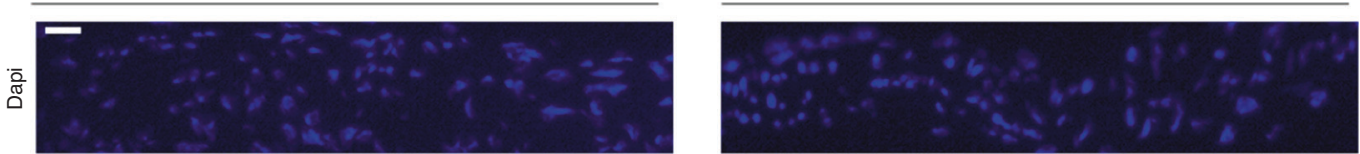

崖

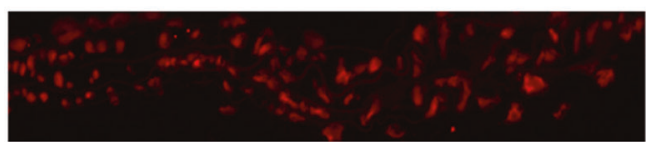

f

Nuclear SMC in Thsd4 +/- (9 months)

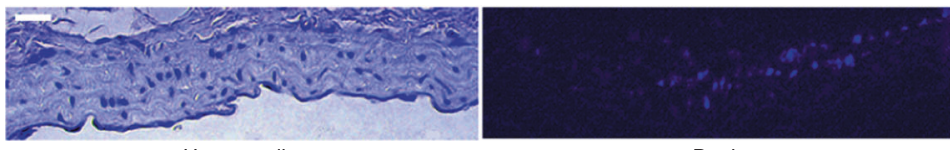

Hematoxylin

Dapi

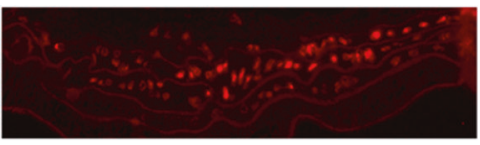

TUNEL

transfected or exogenously supplemented with ADAMTSL6. ${ }^{16}$ It has also been shown that expression of Adamtsl6 rescues microfibril disorder after periodontal ligament injury in a MFS mouse model. ${ }^{36}$ Thus, ADAMTSL6 might constitute a new therapeutic target in TAAD, a group of diseases that still lacks targeted treatment other than appropriate prevention of its life-threatening complications.

Despite the limitations of this study, such as small TAA pedigrees of a complex inherited disorder known for its multifactorial genetic and environmental risk factors, the 
Fig. 4 Thsd4 deficiency induces aortic dilation. Vascular echography analysis was performed to assess thoracic aortic diameters in wild-type (WT) and Thsd $4^{+/-}$mice at different ages (3, 6, 8, and 12 months) and aortic sites (sinus of Valsalva, ascending aorta, arch, and descending aorta). (a) Aortic diameters at different sites are presented in WT and Thsd $4^{+/}$mice at 8 and 12 months. A significant increase in aortic diameter in the Thsd $4^{+/-}$mice $\left(P<1.10^{-4}\right.$ at 6,8 , and 12 months) was observed compared with WT. (b) Aortic diameters at different ages are presented in WT and Thsd $4^{+/-}$mice at Valsalva and descending aorta sites. The aortic diameters significantly increased with time (sinus of Valsalva $P<1.10^{-4}$; ascending aorta $P<1.10^{-4}$; arch $P<1.10^{-3}$; descending aorta $\left.P<1.10^{-2}\right)$ in both WT and Thsd $4^{+-}$mice. A statistically significant interaction between genotype and time was observed at the sinus of Valsalva $(P=0.01)$, arch $(P=0.02)$, and descending aorta $\left(P<1.10^{-3}\right)$, which shows that aortic diameter in Ths $d 4^{+/-}$mice increased more with time than in WT mice. $n=7-17$ mice in each age group for each anatomical site. Black lines represent the mean \pm standard error of the mean. Data were analyzed by two-way analysis of variance (ANOVA). (c, d) Histological analysis revealed that the aortas of Thsd $4^{+/-}$mice at 6 and 9 months showed a patchy accumulation of Alcian Blue-positive areas, along with a more pronounced disorganization of autofluorescent elastin and alteration of extracellular matrix (ECM) compared with a 6-month-old WT mouse. Scale bars, $50 \mu \mathrm{m}$ (c), $30 \mu \mathrm{m}$ (d). (e) Nuclei from Ths $4^{+/-}$mice showed an increased relative ratio of TUNEL-positive to DAPIpositive nuclei, highlighting the increased degradation of DNA in Thsd $4^{+/-}$mice compared with WT mice at the same age. Scale bar, $20 \mu \mathrm{m}$. (f) Comparison of three methods of nuclear visualization in the same aortic region in a 9-month-old Ths $\mathrm{A}^{+/-}$mouse indicated the presence of hematoxylin-positive nuclei (tinctorial staining), whereas most of the nuclei were DAPI-negative (double strand DNA marker) and TUNEL-positive (degraded DNA marker), highlighting the degradation of smooth muscle cell nuclei over time in Thsd $4^{+/}$mice. Scale bar, $20 \mu \mathrm{m}$.

availability of just one sample for histological studies in human, and the analysis of only male heterozygous deficient mice, our work reveals that heterozygous variants in THSD4, encoding ADAMTSL6, play a role in thoracic aortic disease in human. The observed/expected (o/e) score of THSD4, which is the recommended indicator of loss-of-function intolerance in gnomAD, is 0.39 (confidence interval [CI]: 0.28-0.57), indicating that this gene could play a role in human disease.

On the other hand, through the study of 1114 probands and 35 families with a clinical spectrum ranging from syndromic to nonsyndromic TAAD from a French cohort, we found $\sim 35 \%$ of class $4 / 5$ and class 3 variants. This is higher than the $28 \%$ previously reported in a French nonsyndromic heritable TAAD cohort ${ }^{15}$ but can be explained by the presence in our study of syndromic patients. Furthermore, despite the tremendous development in sequencing techniques, $\sim 65 \%$ of the cases remain negative in our collection and that of others. ${ }^{6}$ Therefore, these negative cases constitute a groundwork for other research projects aiming to identify novel genes in hTAAD, of which few are currently under consideration and could be validated in the future.

In conclusion, our findings suggest that pathogenic variants in THSD4 lead to inherited TAAD. This contributes to a better understanding of the pathophysiology of the disease, its genetic diagnosis, prevention, and eventually its treatment.

\section{SUPPLEMENTARY INFORMATION}

The online version of this article (https://doi.org/10.1038/s41436020-00947-4) contains supplementary material, which is available to authorized users.

\section{ACKNOWLEDGEMENTS}

We thank the patients and family members for participating in the study. We thank G. Nicolas, C. Deschildre, and A. Nicoletti (INSERM U1149 and U1148, Paris, France). This research was supported by grants from Leducq Foundation (FLQ 13CVD03), Programme Hospitalier de Recherche Clinique (AOM10108 and CRC 15014), Agence Nationale de la Recherche (NONAGES, ANR14-CE15-0012-01 and GDPM-2, ANR-10-BLAN-1129),
Fédération Française de Cardiologie, Société Française de Cardiologie, Fondation Maladies Rares, Fondation Cœur et Recherche, Conseil de la recherche de I'université Saint-Joseph de Beyrouth and Lebanese National Council for Scientific Research (CNRS-L), the Shriners Hospitals for Children, and the Methusalem grant from the Flemish government and Ghent University (grant number BOF15/MET-V/011).

\section{DISCLOSURE}

The authors declare no conflicts of interest.

Publisher's note Springer Nature remains neutral with regard to jurisdictional claims in published maps and institutional affiliations.

\section{REFERENCES}

1. Howard DPJ, Banerjee A, Fairhead JF, et al. Population-based study of incidence and outcome of acute aortic dissection and premorbid risk factor control: 10-year results from the Oxford Vascular Study. Circulation. 2013;127:2031-2037.

2. Michel J-B, Jondeau G, Milewicz DM. From genetics to response to injury: vascular smooth muscle cells in aneurysms and dissections of the ascending aorta. Cardiovasc Res. 2018:114:578-589.

3. Hofmann Bowman MA, Eagle KA, Milewicz DM. Update on clinical trials of losartan with and without $\beta$-blockers to block aneurysm growth in patients with Marfan syndrome: a review. JAMA Cardiol. 2019:4:702-707.

4. Albornoz G, Coady MA, Roberts M, et al. Familial thoracic aortic aneurysms and dissections-incidence, modes of inheritance, and phenotypic patterns. Ann Thorac Surg. 2006;82:1400-1405.

5. Biddinger A, Rocklin M, Coselli J, Milewicz DM. Familial thoracic aortic dilatations and dissections: a case control study. J Vasc Surg. 1997;25:506-511.

6. Milewicz DM, Regalado E. Heritable thoracic aortic disease overview. In: Adam MP, Ardinger HH, Pagon RA, et al., editors. GeneReviews. Seattle, WA: University of Washington; 1993. http://www.ncbi.nlm.nih.gov/ books/NBK1120/. Accessed 9 February 2018.

7. Aubart M, Gross M-S, Hanna N, et al. The clinical presentation of Marfan syndrome is modulated by expression of wild-type FBN1 allele. Hum Mol Genet. 2015;24:2764-2770.

8. Barbier M, Gross M-S, Aubart M, et al. MFAP5 loss-of-function mutations underscore the involvement of matrix alteration in the pathogenesis of familial thoracic aortic aneurysms and dissections. Am J Hum Genet. 2014;95:736-743.

9. Stheneur C, Collod-Béroud G, Faivre L, et al. Identification of 23 TGFBR2 and 6 TGFBR1 gene mutations and genotype-phenotype investigations in 
457 patients with Marfan syndrome type I and II, Loeys-Dietz syndrome and related disorders. Hum Mutat. 2008;29:E284-E295.

10. Mizuguchi T, Collod-Beroud G, Akiyama T, et al. Heterozygous TGFBR2 mutations in Marfan syndrome. Nat Genet. 2004;36:855-860.

11. Boileau C, Guo D-C, Hanna N, et al. TGFB2 mutations cause familial thoracic aortic aneurysms and dissections associated with mild systemic features of Marfan syndrome. Nat Genet. 2012;44:916-921. https://doi. org/10.1038/ng.2348.

12. Milewicz DM, Trybus KM, Guo D-C, et al. Altered smooth muscle cell force generation as a driver of thoracic aortic aneurysms and dissections. Arterioscler Thromb Vasc Biol. 2017;37:26-34.

13. Milewicz DM, Carlson AA, Regalado ES. Genetic testing in aortic aneurysm disease: PRO. Cardiol Clin. 2010;28:191-197.

14. Renard M, Muiño-Mosquera L, Manalo EC, et al. Sex, pregnancy and aortic disease in Marfan syndrome. PLoS One. 2017;12:e0181166.

15. Arnaud P, Hanna N, Benarroch L, et al. Genetic diversity and pathogenic variants as possible predictors of severity in a French sample of nonsyndromic heritable thoracic aortic aneurysms and dissections (nshTAAD). Genet Med. 2019;21:2015-2024.

16. Tsutsui $K$, Manabe R, Yamada T, et al. ADAMTSL-6 is a novel extracellular matrix protein that binds to fibrillin-1 and promotes fibrillin-1 fibril formation. J Biol Chem. 2010;285:4870-4882.

17. Hubmacher D, Apte SS. ADAMTS proteins as modulators of microfibril formation and function. Matrix Biol. 2015;47:34-43.

18. Le Goff C, Cormier-Daire V. The ADAMTS(L) family and human genetic disorders. Hum Mol Genet. 2011;20:R163-R167.

19. Oller J, Méndez-Barbero N, Ruiz EJ, et al. Nitric oxide mediates aortic disease in mice deficient in the metalloprotease Adamts 1 and in a mouse model of Marfan syndrome. Nat Med. 2017;23:200-212.

20. Fava M, Barallobre-Barreiro J, Mayr U, et al. Role of ADAMTS-5 in aortic dilatation and extracellular matrix remodeling. Arterioscler Thromb Vasc Biol. 2018:38:1537-1548.

21. Ren $P$, Hughes $M$, Krishnamoorthy $S$, et al. Critical role of ADAMTS-4 in the development of sporadic aortic aneurysm and dissection in mice. Sci Rep. 2017;7:12351.

22. Kessler K, Borges LF, Ho-Tin-Noé B, Jondeau G, Michel J-B, Vranckx R. Angiogenesis and remodelling in human thoracic aortic aneurysms. Cardiovasc Res. 2014;104:147-159.

23. Gomez D, Al Haj Zen A, Borges LF, et al. Syndromic and nonsyndromic aneurysms of the human ascending aorta share activation of the Smad2 pathway. J Pathol. 2009;218:131-142.

24. Sakai LY, Keene DR, Renard M, De Backer J. FBN1: the disease-causing gene for Marfan syndrome and other genetic disorders. Gene.2016;591:279-291.

25. Charbonneau NL, Manalo EC, Tufa SF, et al. Fibrillin-1 in the vasculature: in vivo accumulation of eGFP-tagged Fibrillin-1 in a knockin mouse model. Anat Rec (Hoboken). 2020;303:1590-1603.

26. Borges LF, Gomez D, Quintana M, et al. Fibrinolytic activity is associated with presence of cystic medial degeneration in aneurysms of the ascending aorta. Histopathology. 2010;57:917-932.

27. Cikach FS, Koch CD, Mead TJ, et al. Massive aggrecan and versican accumulation in thoracic aortic aneurysm and dissection. JCl Insight. 2018;3:e97167.

28. Mead TJ, Apte SS. ADAMTS proteins in human disorders. Matrix Biol. 2018;71-72:225-239.
29. Le Goff C, Morice-Picard F, Dagoneau N, et al. ADAMTSL2 mutations in geleophysic dysplasia demonstrate a role for ADAMTS-like proteins in TGF-beta bioavailability regulation. Nat Genet. 2008;40:1119-1123.

30. Ahram D, Sato TS, Kohilan A, et al. A homozygous mutation in ADAMTSL4 causes autosomal-recessive isolated ectopia lentis. Am J Hum Genet. 2009;84:274-278.

31. Legare JM, Modaff P, Strom SP, Pauli RM, Bartlett HL. Geleophysic dysplasia: 48 year clinical update with emphasis on cardiac care. Am J Med Genet A. 2018;176:2237-2242.

32. Sabatier L, Djokic J, Fagotto-Kaufmann C, et al. Complex contributions of fibronectin to initiation and maturation of microfibrils. Biochem J. 2013:456:283-295.

33. Hubmacher D, Apte SS. Genetic and functional linkage between ADAMTS superfamily proteins and fibrillin-1: a novel mechanism influencing microfibril assembly and function. Cell Mol Life Sci. 2011;68:3137-3148.

34. Le Goff $C$, Mahaut C, Wang LW, et al. Mutations in the TGF $\beta$ bindingprotein-like domain 5 of FBN1 are responsible for acromicric and geleophysic dysplasias. Am J Hum Genet. 2011:89:7-14.

35. Le Goff C, Cormier-Daire V. From tall to short: the role of TGF $\beta$ signaling in growth and its disorders. Am J Med Genet C Semin Med Genet. 2012;160C:145-153

36. Saito $M$, Kurokawa $M$, Oda $M$, et al. ADAMTSL6 $\beta$ protein rescues fibrillin-1 microfibril disorder in a Marfan syndrome mouse model through the promotion of fibrillin-1 assembly. J Biol Chem. 2011:286:38602-38613

37. Milewicz DM, Michael K, Fisher N, Coselli JS, Markello T, Biddinger A. Fibrillin-1 (FBN1) mutations in patients with thoracic aortic aneurysms. Circulation. 1996;94:2708-2711.

38. Takeda N, Yagi $\mathrm{H}$, Hara $\mathrm{H}$, et al. Pathophysiology and management of cardiovascular manifestations in Marfan and Loeys-Dietz syndromes. Int Heart J. 2016:57:271-277.

39. Kinsey R, Williamson MR, Chaudhry $S$, et al. Fibrillin-1 microfibril deposition is dependent on fibronectin assembly. J Cell Sci. 2008;121:2696-2704.

40. Sabatier $L$, Chen $D$, Fagotto-Kaufmann $C$, et al. Fibrillin assembly requires fibronectin. Mol Biol Cell. 2009:20:846-858.

Open Access This article is licensed under a Creative Commons Attribution-NonCommercial-NoDerivatives 4.0 International License, which permits any non-commercial use, sharing, distribution and reproduction in any medium or format, as long as you give appropriate credit to the original author(s) and the source, and provide a link to the Creative Commons license. You do not have permission under this license to share adapted material derived from this article or parts of it. The images or other third party material in this article are included in the article's Creative Commons license, unless indicated otherwise in a credit line to the material. If material is not included in the article's Creative Commons license and your intended use is not permitted by statutory regulation or exceeds the permitted use, you will need to obtain permission directly from the copyright holder. To view a copy of this license, visit http://creativecommons.org/licenses/by-nc-nd/4.0/.

(C) The Author(s) 2020 